\title{
Effect of feeding ratios on the structure and electrochemical performance of graphite oxide/polypyrrole nanocomposites
}

\author{
HAN YongQin, DING Bing \& ZHANG XiaoGang* \\ College of Material Science \& Engineering, Nanjing University of Aeronautics and Astronautics, Nanjing 210016, China
}

Received February 18, 2011; accepted April 13, 2011

\begin{abstract}
Graphite oxide (GO)/polypyrrole (PPy) nanocomposites (GPYs) were synthesized using in situ polymerization. The effect of the feeding ratios of pyrrole and GO on the structure and electrochemical performances of GPYs was investigated. The structure was characterized via Fourier-transform infrared spectroscopy, scanning electron microscopy, transmission electron microscopy and $\mathrm{X}$-ray diffraction. The electrochemical performance was characterized via cyclic voltammetry, galvanostatic charge-discharge and electrochemical impedance spectroscopy. The results indicate that the more pyrrole is added to GO (with GO concentrations of $20 \%$ and 50\%), the more agglomeration of both PPy and GO layers occurs. This is detrimental to the capacitance utilization of PPy. When the feeding ratio of GO: pyrrole is 80:20, PPys with nanofibrils are dispersed homogenously in/on the exfoliated layer of GO and the conductivity is enhanced. The capacitance utilization of PPy in a composite with a GO concentration of $80 \%$ ( $383 \mathrm{~F} / \mathrm{g}$ ) is higher than that of pure PPy (201 F/g), which indicates the presence of a synergistic effect between GO and PPy.
\end{abstract}

graphite oxide, polypyrrole, nanocomposites, electrochemical performance

Citation: Han Y Q, Ding B, Zhang X G. Effect of feeding ratios on the structure and electrochemical performance of graphite oxide/polypyrrole nanocomposites. Chinese Sci Bull, 2011, 56: 2846-2852, doi: 10.1007/s11434-011-4646-1

Graphite oxide (GO) is a lamellar material which can be exfoliated into the graphene oxide by applying mechanical energy in highly polar solvents, such as water [1]. Recently, GO has received rapidly growing interest because of its unique structure and properties. GO sheets possess a number of hydroxyl and epoxide functional groups anchored onto the surface $s p^{3}$-hybridized carbon atoms. Moreover, they have considerable amounts of $s p^{2}$-hybridized carbon atom-containing carboxyl and carbonyl groups at their sheet edges. Thus, they can be readily dispersed in water [2-6]. Meanwhile, these oxygen-containing groups impart the GO sheets strong reactivity with small polar molecules and polymers, which form GO composites.

Micrometer- or nanometer-sized conducting polymers and their composites have attracted great attention primarily because of their potential applications in batteries, sensors, capacitors, and field-emission applications [7-13]. Among

*Corresponding author (email: azhangxg@163.com) the conducting polymers and composites that have been studied, polypyrrole (PPy) and its composites are among the most extensively studied. This is because they possess high electrical conductivity, interesting redox properties and relatively high environmental stability. Furthermore, PPy is easy to prepare via chemical or electrochemical processes. The electronegative groups of GO, especially carboxyl and hydroxyl groups, can act as the "active sites" for the polymerization of pyrrole [14]. The morphology of the obtained polymers is an oriented nanostructure, such as nanofibers or nanowires. Nanostructured materials often possess a combination of physical and mechanical properties not present in conventional composites, such as electrical conductivity or electrochemical activity. Previous research on GO/PPy nanocomposites (GPYs) has been primarily focused on the improvement of the conductivity and thermal stability of GO [14-17]. Little attention has been given to the effect of feeding ratios on the structure and electrochemical performance of such composites. 
In this paper, GO was synthesized via a modified Hummers method [18] and sonicated for $6 \mathrm{~h}$ to obtain colloidal graphene oxide in aqueous media. GPYs with different mass ratios were prepared via in situ chemical oxidative polymerization in the presence of colloidal graphene oxide. The structure of the composites was characterized via Fourier-transform infrared spectroscopy (FTIR), scanning electron microscopy (SEM), transmission electron microscopy (TEM) and X-ray diffraction (XRD). The electrochemical performance of the composites in $1 \mathrm{~mol} / \mathrm{L} \mathrm{KCl}$ electrolyte was studied via cyclic voltammetry $(\mathrm{CV})$, galvanostatic charge-discharge and electrochemical impedance spectroscopy (EIS).

\section{Experimental}

\subsection{Materials and synthesis}

GO was prepared from natural graphite (325 mesh, Shandong Pingdu Graphite Company, China). Pyrrole (analytical purity, Sigma-Aldrich, St. Louis, MO, USA) was purified via distillation under reduced pressure and stored in a refrigerator prior to use. All other chemicals including ammonium persulfate (APS), $\mathrm{H}_{2} \mathrm{SO}_{4}, \mathrm{KMnO}_{4}, \mathrm{NaNO}_{3}, \mathrm{H}_{2} \mathrm{O}_{2}$ and $\mathrm{KCl}$ (Nanjing Chemical Reagent Company, China) were of analytical grade and used as received without further treatment.

GO was prepared via the modified Hummer's method [18]. The natural graphite powder was oxidized with $\mathrm{KMnO}_{4}$ in concentrated $\mathrm{H}_{2} \mathrm{SO}_{4}$. Ten grams of graphite powder was added to $230 \mathrm{~mL}$ of cold $\left(0^{\circ} \mathrm{C}\right) 98 \% \mathrm{H}_{2} \mathrm{SO}_{4}$. Thirty grams of $\mathrm{KMnO}_{4}$ and $5 \mathrm{~g}$ of $\mathrm{NaNO}_{3}$ were added gradually while stirring and cooling to keep the temperature in the reactor between $0-4{ }^{\circ} \mathrm{C}$. The mixture was then stirred between $0-4^{\circ} \mathrm{C}$ for $1 \mathrm{~d}$. Then it was kept at room temperature for $4 \mathrm{~d}$. Then, $250 \mathrm{~mL}$ of deionized water was slowly added to the mixture. The reaction was terminated by adding $1 \mathrm{~L}$ of deionized water followed by $100 \mathrm{~mL}$ of $5 \% \mathrm{H}_{2} \mathrm{O}_{2}$ solution. The solid product was separated via centrifugation, washed repeatedly with $5 \% \mathrm{HCl}$ solution until the sulfate could not be detected using $\mathrm{BaCl}_{2}$. Then, it was washed 3-4 times with acetone and dried in a vacuum oven at $60^{\circ} \mathrm{C}$ for $24 \mathrm{~h}$.

A $70 \mu \mathrm{L}$ portion of pyrrole monomer $(1 \mathrm{mmol})$ was added to $20 \mathrm{~mL}$ of deionized water and stirred for $30 \mathrm{~min}$. Then, the oxidant APS ( $1 \mathrm{mmol}$ APS was added to $10 \mathrm{~mL}$ deionized water) was added dropwise. The polymerization was allowed to proceed for $12 \mathrm{~h}$ and kept between $0-4^{\circ} \mathrm{C}$. The product that was obtained was filtered and washed with deionized water. Then, it was dried at $60^{\circ} \mathrm{C}$ for $24 \mathrm{~h}$ in vacuum to obtain PPy.

GPYs were prepared via the in situ polymerization of pyrrole in colloidal graphene oxide in an aqueous solution. Nanocomposites with different mass ratios were prepared. Based on their weight feed ratios of GO:pyrrole $(20: 80$, $50: 50$, and 80:20), the resulting composites were designated as GPYs20, GPYs50 and GPYs80. Typically, for GPYs20, $50 \mathrm{mg}$ GO was added into $50 \mathrm{~mL}$ deionized water and sonicated for $6 \mathrm{~h}$ to obtain colloidal graphene oxide. A 209 $\mu \mathrm{L}$ volume of pyrrole monomer was added to the above suspension while it was vigorously stirred. Then $10 \mathrm{~mL}$ of $\operatorname{APS}\left(n_{\mathrm{APS}}: n_{\mathrm{Py}}=1: 1\right)$ aqueous solution was added dropwise. The polymerization was allowed to proceed for $12 \mathrm{~h}$ and was kept between $0-4^{\circ} \mathrm{C}$. The obtained product was filtered and washed with deionized water. Then, it was dried at $60^{\circ} \mathrm{C}$ for $24 \mathrm{~h}$ in vacuum.

\subsection{Characterization}

SEM measurements were carried out using a Hitachi S-4800 scanning electron microscope (Tokyo, Japan). The specimens were platinum-coated prior to examination. TEM measurements were conducted on a JEOL JEM-2100 microscope (Tokyo, Japan). XRD patterns were measured using a Rigaku D/MAX-RC X-ray diffractometer (Tokyo, Japan) with $\mathrm{Cu} \mathrm{K} \alpha$ radiation. FTIR spectra were recorded on a Bruker VECTOR22 FT-IR spectrometer (Karlsruhe, Germany) using pressed $\mathrm{KBr}$ pellets.

All electrochemical experiments were performed on a CHI660c electrochemical work station ( $\mathrm{CH}$ Instruments, Austin, TX, USA) in a three-electrode system. Platinum foils and saturated calomel electrode were used as counter and reference electrodes. The working electrodes were fabricated by mixing electroactive materials (GO, PPy and GPYs), carbon black and polyterafluoroethylene in a mass ratio of $80: 15: 5$ resulting in a homogeneous mixture. The resulting mixture was pressed on a graphite current collector. The electrolyte was $1 \mathrm{~mol} / \mathrm{L} \mathrm{KCl}$. CV tests were done between -0.8 and $0.5 \mathrm{~V}$ at a rate of $10 \mathrm{mV} / \mathrm{s}$. Galvanostatic charge-discharge curves were measured at a current density of $0.5 \mathrm{~A} / \mathrm{g}$. EIS measurements were performed in the frequency range from $10^{5}$ to $0.01 \mathrm{~Hz}$ at an open circuit potential with an ac perturbation of $5 \mathrm{mV}$.

\section{Results and discussion}

\subsection{Morphology and structure of the composites}

SEM and TEM were used to characterize the morphology of the composites. As shown in Figure 1, the SEM image of GO exhibits a layered structure. The GO sheets are folded onto themselves resulting in a stronger absorbance and wrinkled surface. From Figure 1, it can be seen that PPy has globular submicron structure with diameters ranging between 200-300 nm. The surface morphology of the composites was changed by the introduction of PPy. The puckering feature of GO in the composites disappeared compared with that of pure GO. This can be attributed to the agglomeration of GO layers in the composites. The SEM micrographs of GPYs20 and GPYs50 show the larger laminar layers together with the smaller ones. The PPys are not 

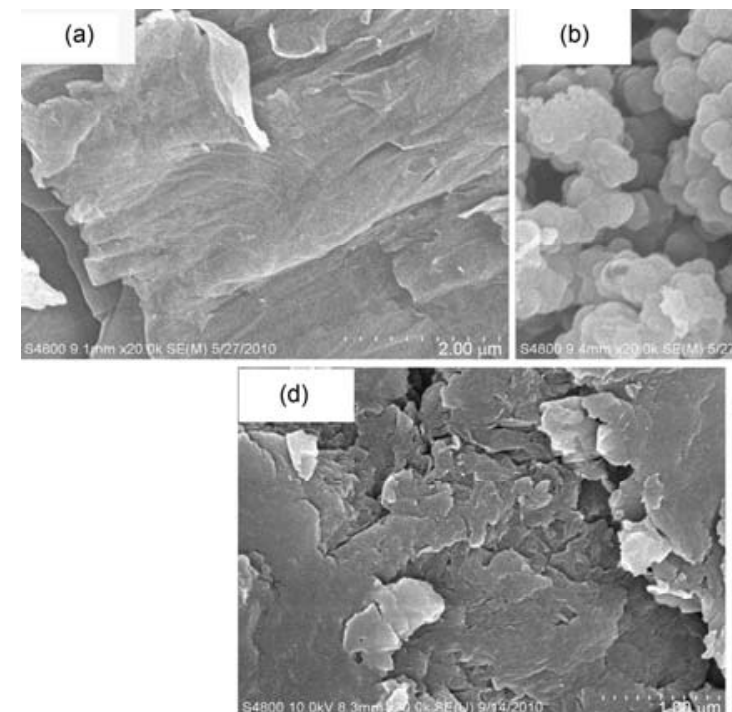

(b)
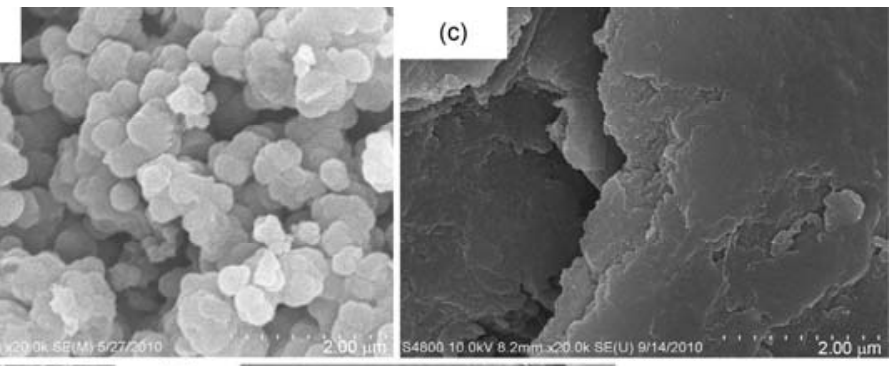

(e)

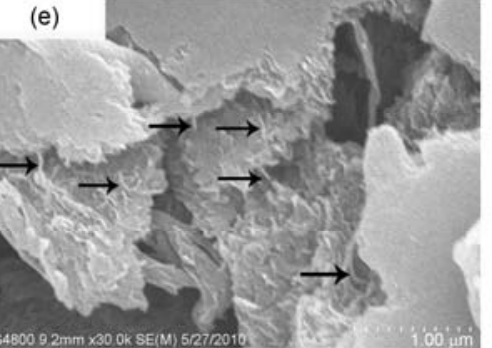

Figure 1 SEM images of GO (a), PPy (b), GPYs20 (c), GPYs50 (d) and GPYs80 (e).

clearly distinguishable in GPYs20 or GPY50. However, in the GPYs80, many PPys with short nanofibrillar morphology and diameters between 10-20 nm can be seen (marked by arrows in Figure 1). GPYs80 may build an improved electron conducting network, which affects the electrochemical performance of the composites.

To confirm the morphology seen in the SEM images, TEM was used to visualize the structure of the nanocomposites. From Figure 2, it can be seen that GO has a crumpled, layered-like structure with a size of tens of micrometers and the PPy has a submicron-spherical morphology with diameters between $200-300 \mathrm{~nm}$, which is in accordance with the SEM observations. Some of PPy spheres were linked with each other, forming microporous structures, which would be responsible for the permeation of the electrolyte within the electrode. For the cases of GPYs20 and GPYs50, the agglomeration of the GO layers can be observed in the composites. Black aggregates for PPy can also be seen on the

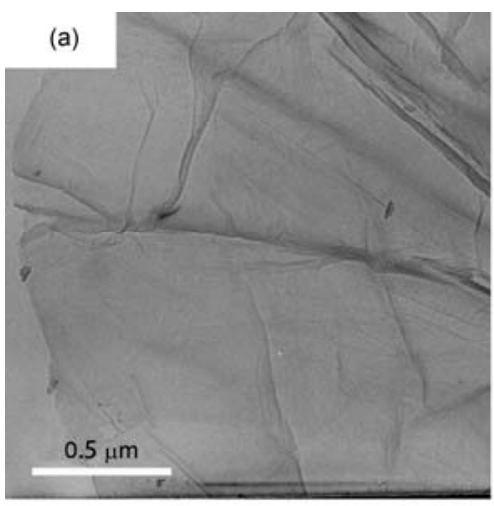

(d)

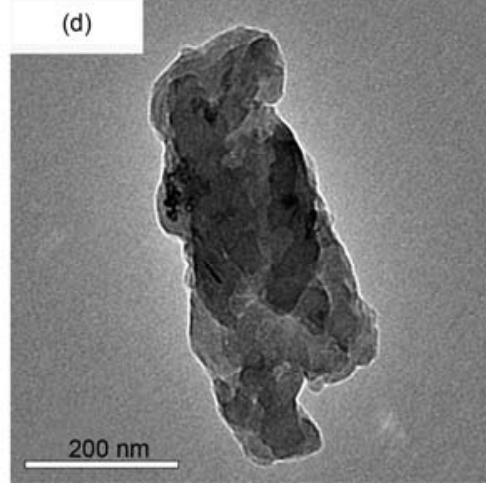

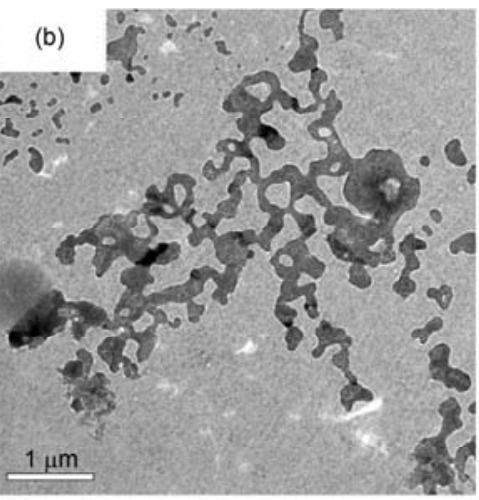

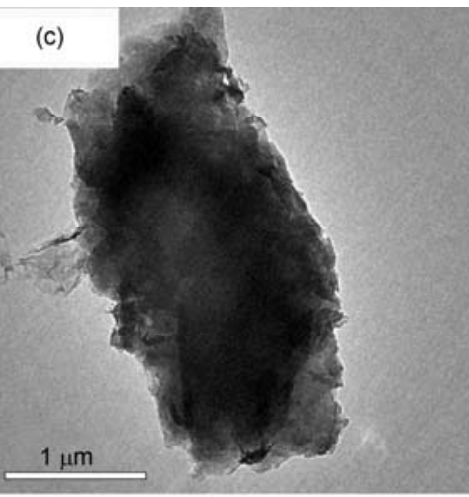

(e)

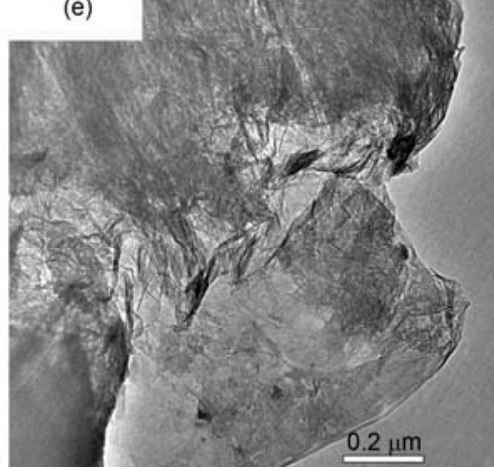

Figure 2 TEM images of GO (a), PPy (b), GPYs20 (c), GPYs50 (d) and GPYs80 (e). 
surface or intercalating between the GO sheets. The morphology of GPYs80 is very different from those of GPYs20 and GPYs50. PPys formed in/on GO layers exhibit uniform dispersed short nanofibers in accordance with the observations from the SEM images $(10-20 \mathrm{~nm})$. The special morphology of the PPys that occurs in GPYs80 suggests that the graphene oxide layers were stably dispersed in the water. Moreover, the addition of pyrrole may have an important contribution to the polymerization of PPy by inducing PPy to grow with an ordered morphology.

The structures of the GO, PPy and the composites were studied via XRD. The results are shown in Figure 3. The characteristic XRD diffraction peak of pure GO sheets appeared at $2 \theta=8.4^{\circ}$. This corresponds to a d-spacing of $1.05 \mathrm{~nm}$, resulting from the diffraction of the (001) plane. This suggests that there is intercalated water in the lamellae of GO, because the GO interlayer distance depends strongly on the GO: $\mathrm{H}_{2} \mathrm{O}$ ratio $[19,20]$. For GPYs 20 and GPYs50, the peaks at $2 \theta=8.4^{\circ}$ shift to $2 \theta=10.4^{\circ}$ and $13.1^{\circ}$, which correspond to interlayer spacings of 0.85 and $0.67 \mathrm{~nm}$. This is because the monolayer of water molecules was separated from the GO layers [21,22]. It can be seen in the XRD pattern of GPYs80 that the characteristic diffraction peak of GO vanished and there is no defined diffraction peak (Figure 3). This indicates that the regular and periodic structure of GO was lost and the GO sheets were delaminated in the composite.

FTIR spectra for GO, PPy and GPYs are shown in Figure 4. The spectrum of GO shows a strong $-\mathrm{OH}$ peak at 3417 $\mathrm{cm}^{-1}$. It also shows other $\mathrm{C}-\mathrm{O}$ functionalities, such as $\mathrm{COOH}$ at $1726 \mathrm{~cm}^{-1}$ and $\mathrm{COC} / \mathrm{COH}$ in the range 1386-1064 $\mathrm{cm}^{-1}$. The FTIR spectrum of synthesized PPy shows absorption bands at 1556, 1471, 1301, 1191, 1041 and $919 \mathrm{~cm}^{-1}$. For GPYs20 and GPYs50, the absorption peaks are similar to those of pure PPy, except that several absorption bands were shifted to lower values. The peak at $1471 \mathrm{~cm}^{-1}$ corresponding to the antisymmetric pyrrole ring-stretching vibrations was downshifted to $1460 \mathrm{~cm}^{-1}$ for

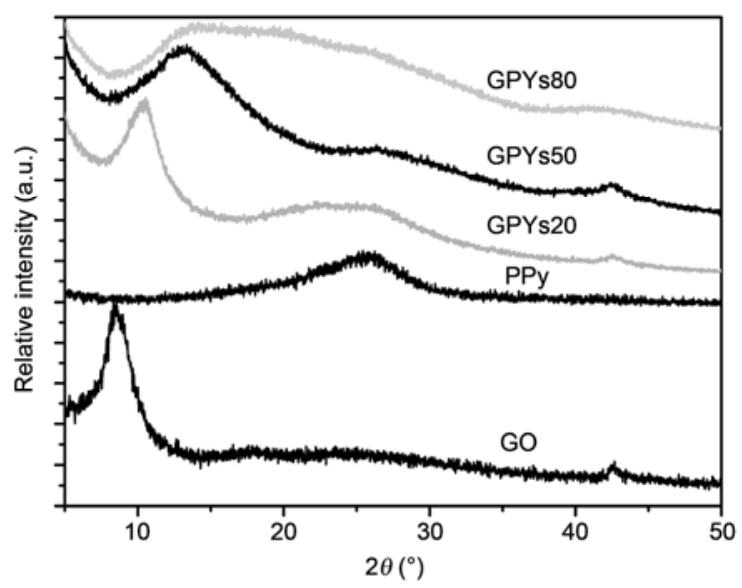

Figure 3 XRD patterns for GO, PPy, GPYs20, GPYs50 and GPYs80.

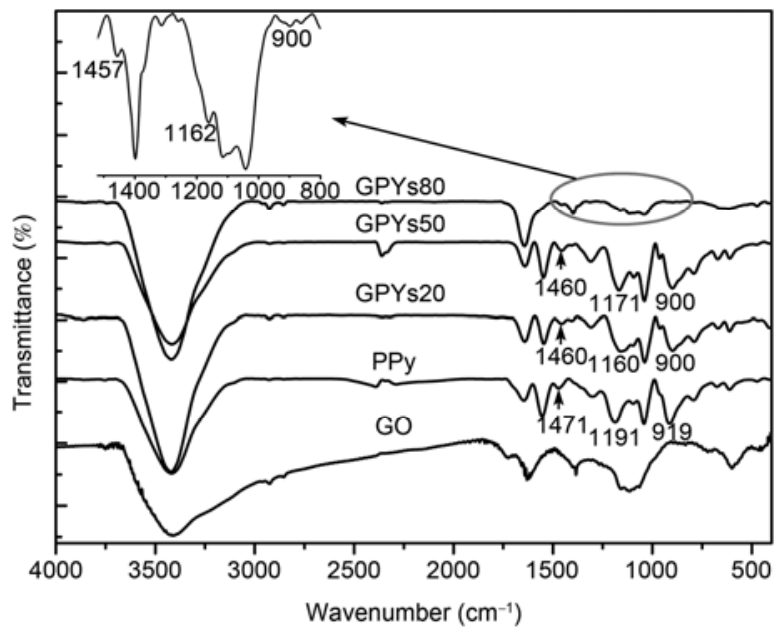

Figure 4 FTIR spectra of GO, PPy, GPYs20, GPYs50 and GPYs80.

GPYs20 and GPYs50 because of the $\pi-\pi$ conjugate effect between the GO layers and PPy. The peak at $1191 \mathrm{~cm}^{-1}$ assigned to the C-N stretching of PPy was also downshifted to $1160 \mathrm{~cm}^{-1}$ for GPYs20 and $1171 \mathrm{~cm}^{-1}$ for GPYs50. This is probably because of the hydrogen bonding between the $\mathrm{C}-\mathrm{OH}$ on the GO layers and nitrogen atoms in PPy. The peak centered at $919 \mathrm{~cm}^{-1}$ can be attributed to the bipolaron state of PPy [23] and was downshifted to $900 \mathrm{~cm}^{-1}$ for all three PPys in GPYs. This shift can be attributed to the increased $\pi$-electron density induced by charge transfer [24]. For the case of GPYs80, although the PPy peaks had weak intensities because of the low content of PPy in the composites, similar downshifts could also be observed as shown in Figure 4 and the magnified area. Analysis of all the above observations indicated the presence of interactions between GO and PPy in the composites.

From the above observations, it can be inferred that the feeding ratios of GO: pyrrole have a significant effect on the structure of the composites. Higher feeding ratios of GO: pyrrole could be beneficial for ordered growth of PPys and the homogenous distribution of GO sheets. A possible formation process for GPYs80 is shown in Figure 5. When sonicated, GO is exfoliated into graphene oxide and can be stably dispersed in water. When a small amount of pyrrole is added to the graphene oxide aqueous solution, the pyrrole cations are not sufficient to occupy the electroactive functional groups of graphene oxide. Thus, graphene oxide can still be stably dispersed in water because of the existence of the remaining electroactive functional groups. This may be favorable for the preparation of PPys with ordered structure and the formation of exfoliated nanocomposites. However, when a large amount of pyrrole is added, the electrostatic attractions between pyrrole cations and electronegative functional groups may reduce the interactions between $\mathrm{GO}$ and $\mathrm{H}_{2} \mathrm{O}$. As a result, the graphene oxide sheets may agglomerate and the layer distance between GO layers will be reduced. 

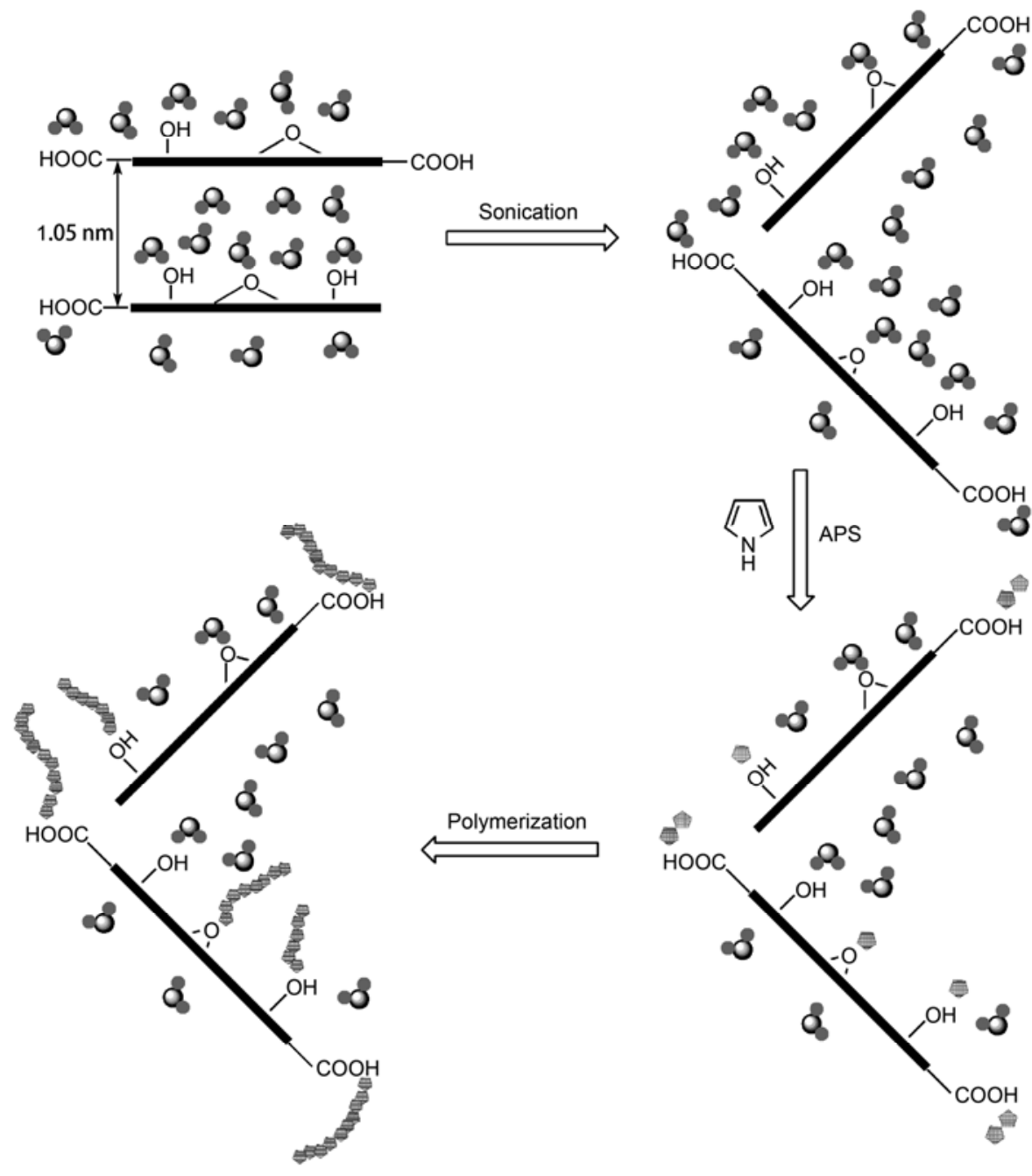

Polymerization
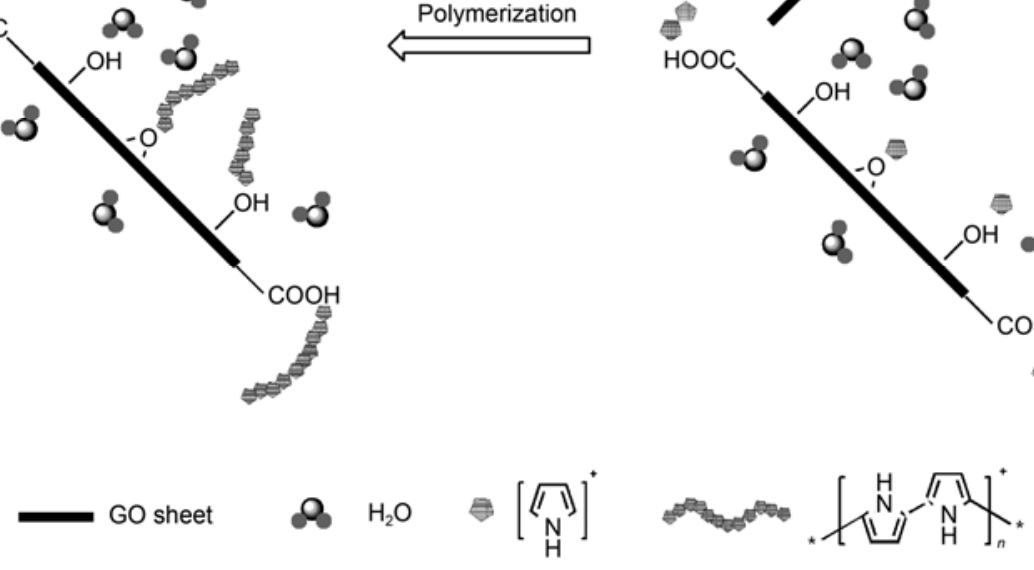

Figure 5 Schematic diagram of the formation of GPYs80

\subsection{Electrochemical performance of the composites}

$\mathrm{CV}$ spectra were taken to investigate the changes in the electrochemical performance of the pure systems and the composites. Figure 6 compares the electrodes at a scan rate of $10 \mathrm{mV} / \mathrm{s}$ between -0.8 to $0.5 \mathrm{~V}$ in $1 \mathrm{~mol} / \mathrm{L} \mathrm{KCl}$ aqueous media. Among the composites, GPYs 20 and GPYs 80 show typical capacitive behaviors with quasi-rectangular profiles. From the voltammograms, it can be seen that the larger current response of the composites corresponds to a higher specific capacitance than that of pure GO at the same scan rate. The enhanced current in the composites can be attributed to the incorporation of PPy into GO, which results in decreasing the distance for the electron shuttling during the electrochemical reaction.

Figure 7 shows the galvanostatic charge-discharge curves for the prepared samples. The specific capacitance of the

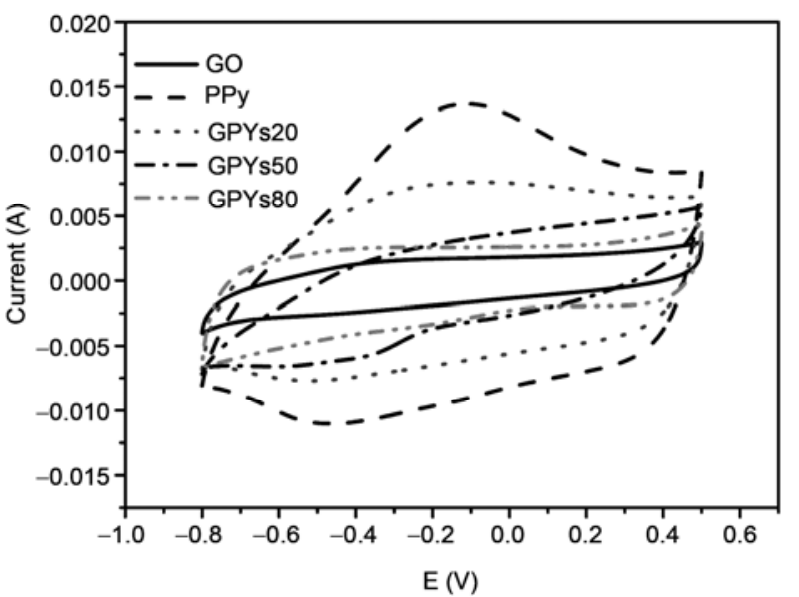

Figure 6 Cyclic voltammograms for GO, PPy, GPYs20, GPYs50 and GPYs 80 in $1 \mathrm{~mol} / \mathrm{L} \mathrm{KCl}$ at a scan rate of $10 \mathrm{mV} / \mathrm{s}$. 


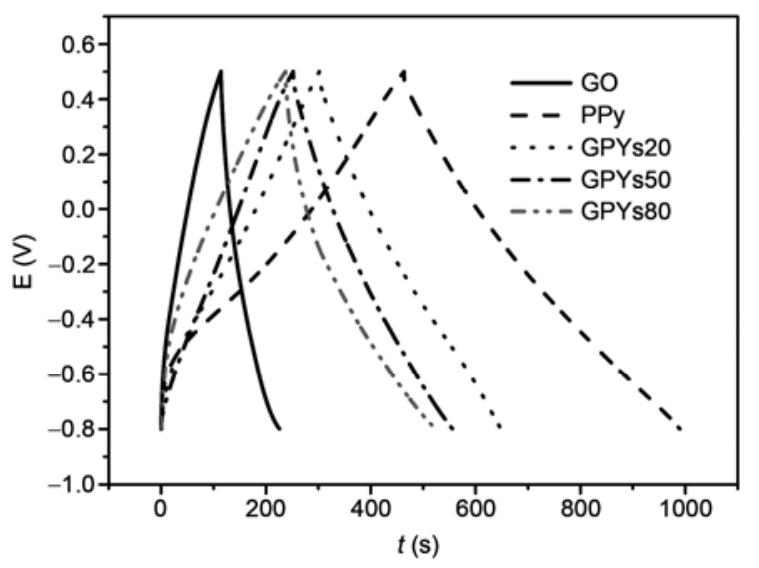

Figure 7 Galvanostatic charge-discharge curve for GO, PPy, GPYs20, GPYs50 and GPYs 80 at a constant current density of $0.5 \mathrm{~A} / \mathrm{g}$.

prepared samples can be obtained from the charge-discharge curves using the following equation

$$
C_{\mathrm{m}}=\frac{Q}{\Delta V \times m}=\frac{I \times t}{\Delta V \times m},
$$

where $I$ is the current during discharge; $t$ is the discharge time; $m$ is the mass of the active material; and $\Delta V$ is the potential drop during the discharge progress [25]. Based on the above equation, the specific capacitances of GO, PPy, GPYs20, GPYs50 and GPYs80 were found to be 43, 201, 133,116 and $111 \mathrm{~F} / \mathrm{g}$, espectively. It is striking that GPYs80 with its limited concentration of PPy (20\%) has relatively good capacitance values $(111 \mathrm{~F} / \mathrm{g})$. By contrast, the capacitance of GO alone is only $43 \mathrm{~F} / \mathrm{g}$. The capacitance utilization of the PPy in the composites $\left(C_{\mathrm{m}, \mathrm{PPy}}\right)$ can be calculated using the following equation

$$
C_{\mathrm{m}, \mathrm{PPy}}=\frac{C_{\mathrm{m}, \text { electrode }}-\left(1-w_{\mathrm{PPy}}\right) C_{\mathrm{m}, \mathrm{GO}}}{w_{\mathrm{PPy}}},
$$

where $C_{\mathrm{m} \text {,electrode }}$ is the specific capacitance of the composite electrode; $C_{\mathrm{m}, \mathrm{GO}}$ is the specific capacitance of the GO electrode; and $w_{\mathrm{PPy}}$ is the weight fraction of PPy in the composites. From eq. (2), the capacitance utilization of PPy in GPYs20, GPYs50 and GPYs80 were found to be 155.5, 189 and $383 \mathrm{~F} / \mathrm{g}$, respectively. From the above results, note that the capacitance utilization of PPy in GPYs20 and GPYs50 is lower than that of pure PPy. The capacitance utilization of PPy in GPYs80 is much higher than that of PPy, indicating a significant synergistic effect from GO and PPy in GPYs80. The feeding ratios have a significant effect on the value of $C_{\mathrm{m}, \mathrm{PPy}}$. Higher feeding ratios for pyrrole/GO lead to the agglomeration of GO and PPy in the composites (GPYs20 and GPYs50). This is supported by the TEM and XRD results. This reduces the contact of PPy with the electrolyte and may be unfavorable for the enhancement of $C_{\mathrm{m}, \mathrm{PPy}}$. However, for the case of GPYs80, the homogeneous disper- sion of nanoscale PPy fibers in/on the GO layers reduces the diffusion and migration length of the electrolyte ions during the fast charge-discharge process and increases $C_{\mathrm{m}, \mathrm{PPy}}$.

EIS is a technique, which is complementary to galvanostatic cycling measurements, which provides more information on the electrochemical frequency behavior of the system. The intersection of the semi-circle with the real axis $\left(\mathrm{Z}^{\prime}\right)$ at high frequencies is a measure of the internal resistance $\left(R_{\mathrm{s}}\right)$ [26]. From Figure 8, it can be seen that at high frequencies the $R_{\mathrm{s}}$ of GO, PPy, GPYs20, GPYs50 and GPYs80 were about 2, 5, 2.8, 1.2, 1.9 and $0.7 \Omega$, respectively. This indicates that the ohmic resistance in the GPYs is smaller than that of pure PPy. The low value for GPYs80 can be attributed to a low ohmic resistance between the electrode and the electrolyte. The diameter of the semi-circle along the real axis gives the charge transfer resistance. Note that the charge transfer resistance of pure PPy is much larger than that of the composites. For GPYs80, the semicircle at high frequencies is not present, which suggests that interfacial charge-transfer resistance in GYs80 is low, because of its high conductivity. From the above results, it can be further concluded that a higher feeding ratio of GO: pyrrole can enhance the conductivity and the capacitance utilization of the electrochemical performance of PPy in GPYs.

\section{Conclusions}

In summary, GPYs with different weight ratios were synthesized via in situ polymerization. The morphologies of GPYs can be controlled by adjusting the feed ratio of GO to pyrrole. At a GO:pyrrole feeding ratio of 80:20, homogenous PPys with nanofibrillar morphologies situated in/on the exfoliated GO layers were obtained. Our electrochemical studies showed that GPYs80 exhibited a synergistic effect between GO and PPy. The capacitance utilization of PPy in

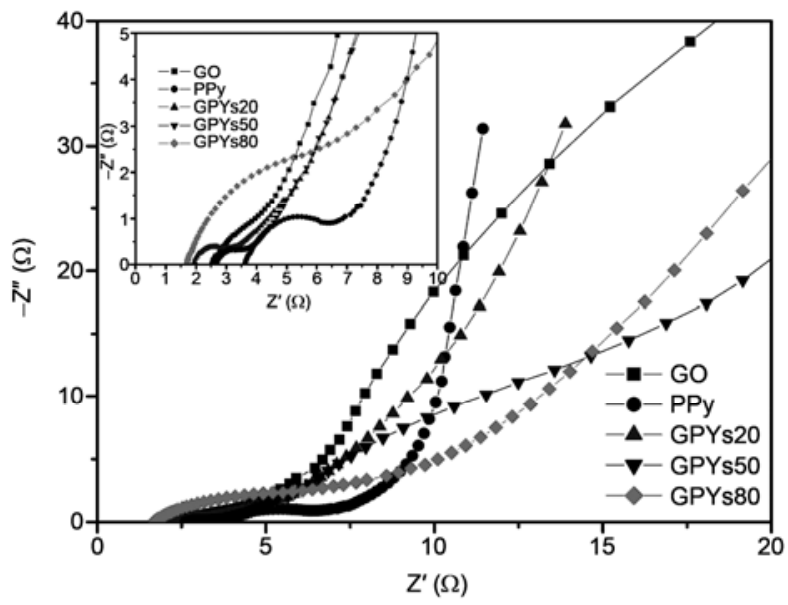

Figure 8 Nyquist plots for GO, PPy, GPYs20, GPYs50 and GPYs80. 
GPYs80 is $383 \mathrm{~F} / \mathrm{g}$, which is higher than that of pure PPy (201 F/g). Our experimental results indicate that the increased addition of PPy to GO may lead to the agglomeration of both GO and PPy and is less suitable for capacitance applications. Moreover, increased concentration of PPy may reduce the ordering of the nanostructures and the homogenous dispersion of PPy and GO layers. This study may serve as a guide for the future study of the relationship between structure and electrochemical performance of GPYs.

This work was supported by the National Key Basic Research Program of China (2007CB209703), the National Natural Science Foundation of China (20633040 and 20873064) and the Postdoctoral Science Foundation of China (20090461108).

1 Park S, Ruoff R S. Chemical methods for production of graphenes. Nat Nanotechnol, 2009, 4: 217-224

$2 \mathrm{He} \mathrm{H}$, Klinowski J, Forster M, et al. A new structural model for graphite oxide. Chem Phys Lett, 1997, 287: 53-56

3 Lerf A, He H, Riedl T, et al. ${ }^{13} \mathrm{C}$ and ${ }^{1} \mathrm{H}$ MAS NMR studies of graphite oxide and its chemically modified derivatives. Solid State Ionics, 1997, 101-103: 857-862

4 Lerf A, He H, Forster M, et al. Structure of graphite oxide revisited. J Phys Chem B, 1998, 102: 4477-4482

5 Hontoria-Lucas C, Lopez-Peinado A J, Lopez-Gonzalez J D, et al. Study of oxygen-containing groups in a series of graphite oxides: Physical and chemical characterization. Carbon, 1995, 33: 1585-1592

6 Szabo T, Tombacz E, Illes E, et al. Enhanced acidity and pH dependent surface charge characterization of successively oxidized graphite oxides. Carbon, 2006, 44: 537-545

7 Nishide H, Oyaizu K. Materials science-Toward flexible batteries. Science, 2008, 319: 737-738

8 Lakard B, Segut O, Lakard S, et al. Potentiometric miniaturized $\mathrm{pH}$ sensors based on polypyrrole films. Sens Actuator B-Chem, 2007, 122: $101-108$

9 Sharma R K, Rastogi A C, Desu S B. Pulse polymerized polypyrrole electrodes for high energy density electrochemical supercapacitor. Electrochem Commun, 2008, 10: 268-272

10 Cheng Y, Zhao Z S, Zhang F, et al. Preparation of $\mathrm{Fe}_{3} \mathrm{O}_{4}-\mathrm{SiO}_{2}-$ polypyrrole core-shell nanoparticles, and their adsorption of $\mathrm{Cr}_{2} \mathrm{O}_{7}^{2-}$.
Chinese Sci Bull, 2010, 55: 2904-2909

11 Huang L Y, Hou W B, Liu Z O, et al. Polypyrrole-coated styrene-butyl acrylate copolymer composite particles with tunable conductivity. Chinese Sci Bull, 2005, 50: 971-975

$12 \mathrm{Li} \mathrm{F}$, Shi J J, In X. Synthesis and supercapacitor characteristics of PANI/CNTs composites. Chinese Sci Bull, 2010, 55: 1100-1106

13 Wang X X, Yang T, Jiao K. Controllable fabrication of Au micro/nanostructures on self-doped polyaniline nanofibers via electrochemical deposition and its application for DNA immobilization. Chinese Sci Bull, 2010, 55: 4125-4131

14 Han Y Q, Lu Y. Characterization and electrical properties of conductive polymer/colloidal graphite oxide nanocomposites. Composite Sci Technol, 2009, 69: 1231-1237

$15 \mathrm{Gu} \mathrm{Z} \mathrm{M,} \mathrm{Li} \mathrm{C} \mathrm{Z,} \mathrm{Wang} \mathrm{G} \mathrm{C,} \mathrm{et} \mathrm{al.} \mathrm{Synthesis} \mathrm{and} \mathrm{characterization} \mathrm{of}$ polypyrrole/graphite oxide composite by in situ emulsion polymerization. J Polym Sci Pol Phys, 2010, 48: 1329-1335

16 Gu Z, Zhang L, Li C. Preparation of highly conductive polypyrrole/graphite oxide composites via in situ polymerization. J Macromol Sci B, 2009, 48: 1093-1102

17 Han Y Q, Lu Y. Preparation and characterization of graphite oxide/ polypyrrole composites. Carbon, 2007, 45: 2394-2399

18 Hummers W S, Offeman R E. Preparation of graphite oxide. J Am Chem Soc, 1958, 80: 1339

19 Nakajima T, Mabuchi A, Hagiwara R. A new structure model of graphite oxide. Carbon, 1988, 26: 357-361

20 Kovtyukhova N I, Ollivier P J, Martin B R, et al. Layer-by-layer assembly of ultrathin composite films from micron-sized graphite oxide sheets and polycations. Chem Mater, 1999, 11: 771-778

21 Liu Z, Wang Z M, Yang X, et al. Intercalation of organic ammonium ions into layered graphite oxide. Langmuir, 2002, 18: 4926-4932

22 Bissessur R, Liu P K Y, Scully S F. Intercalation of polypyrrole into graphite oxide. Synthetic Met, 2006, 156: 1023-1027

23 Liu Y C, Lin Y T. Strategy and characteristics of polypyrrole deposited on silver substrates with silver-containing nanocomplexes. J Phys Chem B, 2003, 107: 11370-11375

24 Zhang W X, Wen X G, Yang S H. Synthesize and characterization of uniform arrays of copper sulfide nanorods coated with nanolayers of polypyrrole. Langmuir, 2003, 19: 4420-4426

25 Jiang J H, Kucernak A. Electrochemical supercapacitor material based on manganese oxide: Preparation and characterization. Electrochim Acta, 2002, 47: 2381-2386

26 Kalpana D. New, low-cost, high-power poly(o-anisidine-co-metanilic acid)/activated carbon electrode for electrochemical supercapacitors. J Power Sources, 2009, 190: 587-591

Open Access This article is distributed under the terms of the Creative Commons Attribution License which permits any use, distribution, and reproduction in any medium, provided the original author(s) and source are credited. 